\title{
Positive solutions of an elliptic equation with strongly nonlinear lower order terms
}

\author{
FRANCOIS DE THELIN
}

\begin{abstract}
In this paper we study the existence of positive solutions of the equation:

$$
\Delta_{p} u+g(x, u)=0
$$
\end{abstract}

in the case when the growth of $g(x,$.$) is allowed to be of exponential type.$

\section{INTRODUCTION}

Let $1<p<+\infty$ and let $\Omega$ be a bounded regular open set in $\mathbb{R}^{N}$. We look for positive solutions, $u \in \mathrm{W}_{0}^{1, p}(\Omega)$, of the equation:

$$
\Delta_{p} u+g(x, u)=0 \quad \text { in } \quad \Omega
$$

where $F(\nabla u)=|\nabla u|^{p-2} \nabla u$ and $\Delta_{\mathrm{p}} u=\operatorname{div} F(\nabla u)$.

We are specially interested by the case when the growth of $g$ near

$u=+\infty$ is not of polynomial type, for example of exponential type.

In the case when $\Omega$ is a starshaped domain and $g(x, u)=|u| r-2 u$ with $\gamma(N-p)>N p$, it is well known $[7,9,10,12,13]$ that (E) cannot have positive solutions $u \in \mathrm{W}_{0}^{\text {,p }}(\Omega)$.

On the other hand, in the case when $p=2$ and $\Omega=A=\left\{x \in \mathbb{R}^{\mathrm{N}}: \rho<|x|<\right.$ $R$ \} with $0<\rho<R<+\infty$, recent papers have shown that (E) has positive solutions:

- either for $g(u)=0\left(u^{k}\right), k>-1$, near $u=+\infty[3]$;

- or for $R-\rho$ sufficiently small [2].

In this paper, proving that radially symmetric functions in $\mathrm{W}_{0}^{1, p}(A)$ are in $L^{\infty}(A)$, we can obtain positive solutions of $(E)$ for any $\left.p \in\right] 1$, $+\infty[$, any $R-\rho>0$, and any growth of $g$ near $u=+\infty$. 
In the limit case $N=p, \mathrm{~W}_{0}^{1, p}(\Omega) \not \subset \mathrm{L}^{\infty}(\Omega)$ but [1] $\mathrm{W}_{0}^{1, p}(\Omega) \subset L_{M}(\Omega)$, where $L_{M}$ is the Orlicz space associated with the Young function:

$$
\mathrm{M}(\zeta)=\exp \left(|\zeta| \mathrm{p}^{*}-1\right), \frac{1}{p}+\frac{1}{p^{*}}=1
$$

Trudinger [15] has shown that for $p=2$, any $q \in] 0,2[$ and any $c>0$, there are some $\lambda>0$ and $u \in \mathrm{W}_{0}^{1,2}(\Omega)$ such that:

$$
\Delta u+\lambda \mathcal{u}^{\alpha} \exp \left(\mid u^{\mid}\right)=0 \text { and } \int_{\Omega} \int_{0}^{u(x)} t^{\sigma} \exp \left(t^{a}\right) d t d x=c
$$

In this paper we extend these results to $p \neq 2$ and eliminate this $\lambda$.

The particular case $N=p, \Omega=B(0, R)$ is interesting because we can prove that, for any growth of $g$ near $u=+\infty,(\mathrm{E})$ can have positive radially symmetric solutions if $R$ is sufficiently large; we extend to the case $p \neq 2$ the results of Hempel $[4,5]$ and Nehari [6].

As a conclusion, consider the example:

$$
g(x, \zeta)=|\zeta|^{\sigma} \exp \left(|\zeta|^{a}\right) \text { with } \sigma>p-1 \text { and } q>0
$$

(E) has positive solutions:

- for $p>N$ or $\Omega=A$

(Theorem 1)

- for $p=N$ and $q<p^{*}$

(Theorem 2)

- or $p=N, \Omega=B(0, R), R>R_{\omega}$ $\sigma>\max (p-1,1)$ and $q>1$

(Theorem 3)

\section{BOUNDED SOLUTIONS}

Let $X$ be a closed subspace of $\mathrm{W}_{0}^{1, p}(\Omega) . g$ is asumed to be a Caratheodory function satisfying the following conditions:

$$
\begin{aligned}
& \forall x \in \Omega, \forall \zeta \in \mathbb{R}, g(x, \zeta) \geqslant 0 \\
& \text { and } \forall x \in \Omega, \forall \zeta>0, g(x, \zeta)>0
\end{aligned}
$$

(H2) $\quad \forall K>0, \exists M>0$ such that for any $\zeta \in \mathbb{R},|\zeta| \leqslant K$, and for any $x \in \Omega, g(x, \zeta) \leqslant M$;

(H3) There exist some $\sigma_{0}>p-1$ and $\zeta_{0} \geqslant 0$ such that: 


$$
\begin{aligned}
& \forall \zeta \geqslant \zeta_{0}, \zeta \rightarrow \frac{G(x, \zeta)}{\zeta_{0}-\zeta} \text { is a non decreasing function } \\
& \text { where } G(x, \zeta)=\int_{0}^{\zeta} g(x, s) d s
\end{aligned}
$$

Remark: It is sufficient to suppose that $g$ satisfies $(\mathrm{H} 1)$ and $(\mathrm{H} 2)$ on $\mathbb{R}_{+}$; it can be easily extended to a function satisfying $(\mathrm{H} 1)$ and $(\mathrm{H} 2)$ on $\mathbb{R}$.

\section{Theorem 1:}

Let $g$ satisfy the conditions $(H I),(H 2),(H 3)$ and suppose that:

(i) $X \subset L^{\infty}(\Omega)$

(ii) There exist some $\zeta_{1}>0, \sigma_{1}>p-1$ and $c>0$ such that:

$$
\forall x \in \Omega, \forall \zeta \in\left[0, \zeta_{J}\right], G(x, \zeta) \leqslant c \zeta_{1}^{\sigma_{1}+1}
$$

Then there is at least one positive solution $u \in X \cap C^{t, a}(\Omega)$ of $(E)$.

The condition ( $i$ ) is satisfied for $X=\mathrm{W}_{0}^{1, p}(\Omega)$ and any bounded open set $\Omega$ in $\mathbb{R}^{N}$ in the case when $p>N$; the following proposition gives an other interesting example.

\section{Proposition 1:}

Let $0<\rho<R<+\infty$ and $\Omega$ be an annulus in $\mathbb{R}^{N}$ :

$\Omega=\left\{x \in \mathbb{R}^{N}: \rho<|x|<R\right\}$. Let $X$ be the set of radially symmetric functions in $W_{0}^{\text {I.p }}(\Omega)$.

Then, there exist a positive constant $C(N, p, \rho, R)$ such that:

$$
\forall u \in X, \forall x \in \Omega,|u(x)| \leqslant C(N, p, p, R)\|\nabla u\|_{p}
$$

\section{Examples:}

Let $h: \mathbb{R}_{+} \rightarrow \mathbb{R}_{+}$be a positive non decreasing continuous function and $g(x, \zeta)=\zeta^{\sigma} h(\zeta)$ where $\sigma>p-1$; for instance $g(x, \zeta)=\zeta^{\circ} \exp (\zeta), \sigma>p-1$ $q>0$; then $g$ satisfies (H1), (H2), (H3) and (ii). of

In the case when $\Omega$ is an annulus and $\sigma>1$, we obtain positive solutions 


$$
\Delta u+u^{\sigma} h(u)=0 \text { in } \Omega
$$

without any limiting condition as $g(u)=0\left(u^{k}\right)$ when $u \rightarrow+\infty$ (GARAIZAR [3]), neither $R$ - $\rho$ small (BANDLE - PELETIER [2]); besides we obtain analogous results for $p \neq 2$ and $\sigma>p-1$. On the other hand our conditions are more restrictive than [2], [3] on the growth of $g$ and on the limit of $g(u)$ when $u \rightarrow 0$.

\section{Proof of Proposition 1}

Let $u(x)=\varphi(|x|)$; we have

$$
-\varphi(|x|)=\varphi(R)-\varphi(|x|)=\int_{|x|}^{R} \varphi^{\circ}(t) d t
$$

By Hölder's inequality we get:

$$
\begin{aligned}
& |u(x)| \leqslant\left(\left.\int_{|x|}^{R}\left|\varphi^{*}(t)\right| \nabla t^{\mathrm{N}-1} d t\right|^{1 / p}\left|\int_{|x|}^{R} \frac{d t}{t^{(\mathrm{N}-1) /(p-1)}}\right|^{1 / p^{*}}\right. \\
& \int_{|x|}^{R}\left|\varphi^{*}(\mathrm{t})\right| \mathrm{p} t^{\mathrm{N}-1} d t=\left.\frac{1}{\omega_{\mathrm{N}}}\right|_{|x| \leqslant|y| \leqslant R} \mid \nabla u(y)^{\mid p} d y
\end{aligned}
$$

whence the result with:

$$
C(N, p, \rho, R)=\left.\frac{I}{\omega_{N}^{1 / p}} \iint_{\rho}^{R} \frac{d t}{t^{(N-1) /(p-1)}}\right|^{\frac{1}{p^{*}}}
$$

The proof of Theorem 1 needs the following lemmas.

\section{Lemma 1:}

For any $u \in X$, let us consider:

$$
J(u)=\frac{1}{p} \int_{\Omega} \mid \nabla u(x)^{p} d x-\int_{\Omega} G(x, u(x)) d x
$$

Suppose that $g$ satisfies $(H I),(H 2),(H 3)$. Then any sequence $\left(u_{j}\right) \subset X$ such $\left|J\left(u_{j}\right)\right| \leqslant K$ and $J^{\prime}\left(u_{j}\right) \rightarrow O$ in $X$, is bounded in $X$. 


\section{Proof:}

For any $v \in X$, we have:

$$
J^{\prime}(u)(v)=\int_{\Omega} F(\nabla u) \cdot \nabla v-\int_{\Omega} g(., u) v
$$

$\Omega$ being a bounded set we set:

$$
\left.\|u\|_{X}=\|\nabla u\|_{p}=\iint_{\Omega}|\nabla u|^{p}\right)^{\frac{1}{p}}
$$

Suppose that a subsequence denoted by $u_{j}$ be such that $\lim \left\|u_{j}\right\|_{x}=+\infty$; we get:

$$
\begin{aligned}
& -\frac{K}{\left\|u_{j}\right\|_{x}^{p}} \leqslant \frac{1}{p}-\frac{\int_{\Omega}^{\prime} G\left(., u_{j}\right)}{\left\|u_{j}\right\|_{x}} \leqslant \frac{K}{\left\|u_{j}\right\|_{x}^{p}} \\
& -\frac{\varepsilon}{\left\|u_{j}\right\|_{x}^{p-1}} \leqslant 1-\frac{\int_{\Omega} g\left(., u_{j}\right)}{\left\|u_{j}\right\|_{x}^{p}} \leqslant \frac{\varepsilon}{\left\|u_{j}\right\|_{x}^{-1}}
\end{aligned}
$$

whence $\lim _{j \rightarrow+\infty} \frac{\int_{\Omega}^{\mathrm{G}\left(., u_{j}\right)}}{\int_{\Omega} u_{j} g\left(., u_{j}\right)}=\frac{1}{p}$

(H3) gives for any $\zeta \geqslant \zeta_{0}: \zeta g(., \zeta) \geqslant\left(\sigma_{0}+1\right) G(., \zeta)$, whence:

$$
\begin{gathered}
\iint_{\Omega}^{G\left(., u_{j}\right) \leqslant C_{1}+\frac{1}{\left(\sigma_{0}+1\right)} \int_{\Omega} u_{j} g\left(., u_{j}\right)} \\
\lim _{j \rightarrow+\infty} \frac{\int_{\Omega} G\left(., u_{j}\right)}{\int_{\Omega} u_{j} g\left(, u_{j}\right)} \leqslant \frac{1}{\sigma_{0}+1}<\frac{1}{p}
\end{gathered}
$$


A contradiction, whence $\left\|u_{j}\right\|_{x}$ is bounded.

\section{Lemma 2:}

If the hypothesis of Theorem 1 are satisfied, $J \in C^{\prime}(X)$ and satisfies the Palais - Smale condition.

\section{Proof:}

An easy conseqence of Lebesgue's theorem shows that for $u_{j} \rightarrow u$, $\lim _{j \rightarrow+\infty} \| g\left(., u_{j}\right)-\left(g(., u) \|_{p^{*}}=0\right.$, whence $J \in C^{\prime}(\mathrm{X})$.

Suppose that $\left|J\left(u_{j}\right)\right| \leqslant K$ and $J^{\prime}\left(u_{j}\right) \rightarrow 0 ;$ by lemma $1, \mathrm{~g}\left(, u_{j}\right)$ is bounded, and the injection $X \subset L^{p}$ being compact, there exists a subsequence denoted by $u_{j}$ which converges to $u$ in strong $L^{p}$.

So, $\lim _{n, m \rightarrow+\infty} I_{n, m}=0$ where

$$
\begin{gathered}
I_{n, m}=\int_{\Omega}\left[F\left(\nabla u_{n}\right)-F\left(\nabla u_{m}\right)\right] \cdot \nabla\left(u_{n}-u_{m}\right) \\
=\left(J^{\prime}\left(u_{n}\right)-J^{\prime}\left(u_{m}\right)\right)\left(u_{n}-u_{m}\right)+\int_{\Omega}\left[g\left(., u_{n}\right)-g\left(, u_{m}\right)\right]\left(u_{n}-u_{m}\right) .
\end{gathered}
$$

On the other hand we have:

$$
\left\|\nabla u_{n}-\nabla u_{m}\right\|_{p}^{p} \leqslant c\left\{I_{n, m}\right\} \frac{\alpha}{2}\left\{\left\|\nabla u_{n}\right\|_{p}^{p}+\left\|\nabla u_{m}\right\|_{p}^{p}\right\} 1-\frac{\alpha}{2}
$$

where $\alpha=\min (p, 2)$ (for example see [11]).

Whence $u_{j}$ converges to $u$ in $X$; the Palais-Smale condition is satisfied.

\section{Proof of Theorem 1:}

We shall apply Pass-Mountain Lemma [8] to the function $J$ defined in Lemma $1 . J$ satisfies Palais-Smale condition and $J(0)=0$.

Let us show that, for $\|u\|_{x}=r$ sufficiently small, we have $J(u) \geqslant \alpha>0$. By

(i) there is some $c^{\prime}>0$ such that,

$$
\begin{gathered}
\forall x \in \Omega,|u(x)| \leqslant c^{\prime}\|u\|_{x} ; \text { for }\|u\|_{x} \leqslant \frac{\zeta_{1}}{c^{\prime}} \text { we obtain with (ii): } \\
G(x, u(x)) \leqslant c|u(x)|^{\sigma_{1}+1} \leqslant c(c)^{\sigma_{1}+1}\|u\|_{x^{\sigma_{1}+1}}
\end{gathered}
$$




$$
J(u) \geqslant \frac{1}{p}\|u\|_{x}^{p}\left[1-c^{\prime \prime}\|u\|_{x}^{\sigma_{1}+1-p}\right]
$$

For $\|u\|_{x}=r \leqslant \min \left[\frac{\zeta_{1}}{c^{r}}, \frac{1}{2 c^{\prime \prime}}\right]$ we get $J(u) \geqslant \frac{r^{p}}{2 p}=\alpha>0$.

Now, let us consider $\mathcal{u}_{0} \in X$ such that:

$$
\forall x \in \Omega_{0}, u_{0}(x) \geqslant a_{0}>0 \text { and meas }\left(\Omega_{\mathrm{o}}\right)>0 .
$$

For $\lambda$ sufficiently large, $\lambda \alpha_{0} \geqslant \zeta_{0}$ and by (H3):

$$
\int_{\Omega} G\left(., \lambda u_{0}\right) \geqslant \int_{\Omega_{0}} G\left(, \lambda u_{0}\right) \geqslant \beta \lambda^{s_{0}+1}
$$

where $\beta=\frac{1}{\zeta_{0}^{\sigma_{0}+1}} \int_{\Omega_{0}} G\left(x, \zeta_{0}\right)\left|u_{0}(x)\right|^{\sigma_{0}+1} d x>0$

We then obtain

$$
\lim _{\lambda \rightarrow+\infty} J\left(\lambda u_{0}\right) \leqslant \lim _{\lambda \rightarrow+\infty}\left[\frac{\lambda^{p}}{p}\left\|u_{0}\right\|_{\boldsymbol{\chi}}-\beta \lambda^{\sigma_{0}+1}\right]=-\infty
$$

and there is some $v_{0} \in X, v_{0} \neq 0$, such that $J\left(v_{0}\right)=0$.

By the Pass-Mountain lemma, there exists some $u_{0} \in X, u_{0} \not \equiv 0$, such that $J^{\prime}\left(u_{0}\right)=0$ :

$$
\forall v \in X, \int_{\Omega} F\left(\nabla u_{0}\right) \cdot \nabla v-\int_{\Omega} g\left(., u_{0}\right) v=0 .
$$

By TOLKSDORF's regularity results $u_{0} \in \mathrm{C}^{1, a}(\Omega)$ [14], and by VAZQUEZ's maximum principle [16], $u_{0}>0$ in $\Omega$.

\section{SOLUTIONS IN AN ORLICZ SPACE}

Let us recall that a Young function $M$ is an even convex function from $\mathbb{R}$ to $\mathbb{R}_{+}$, such that:

$$
\lim _{\zeta \rightarrow 0} \frac{M(\zeta)}{\zeta}=0 \text { and } \lim _{\zeta \rightarrow+\infty} \frac{M(\zeta)}{\zeta}=+\infty
$$

The conjugate $M^{*}$ of $M$ is defined by: 


$$
M^{*}(\zeta)=\operatorname{Sup}_{s \in \mathbb{R}}[\zeta s-M(s)]
$$

The Orlicz space $L_{M}(\Omega)$ is the set of measurable functions $u$ defined on $\mathbb{R}$ such that there is some $\lambda>0$ with

$$
\int_{\Omega} M\left(\frac{u}{\hbar}\right)<+\infty \text {. }
$$

$L_{M}(\Omega)$ is a Banach space for the following norm:

$$
\|u\|_{M}=\operatorname{lnf}\left(\lambda>0: \int_{\Omega} M\left(\frac{u}{\hbar}\right) \leqslant 1\right) .
$$

Let $E_{w}(\Omega)$ be the closure of $\mathrm{D}(\Omega)$ in $L_{w}(\Omega)$.

We say that $M$ is superhomogeneous of degree $(\sigma+1)$ if there exists some $K>0$ such that [11]:

$$
\forall \zeta \in \mathbb{R}, \forall h \in[0,1], M(h \zeta) \leqslant h^{\sigma+1} M(K \zeta) .
$$

Let $\Omega$ be a bounded regular open set in $\mathbb{R}^{\mathrm{N}}$.

In the case when $N=p, \mathrm{~W}_{0}^{1, p}(\Omega) \not L^{\infty}(\Omega)$, but $\mathrm{W}_{0}^{1, p}(\Omega) \subset E_{M_{1}}(\Omega)$ [1] where

$$
M_{1}(\zeta)=\exp |\zeta|^{*}-1, \quad \frac{1}{p}+\frac{1}{p^{*}}=1
$$

So, we can get the following Theorem.

\section{Theorem 2:}

Let $g$ satisfy the conditions (H1). (H2), (H3). Suppose that there exists a Young function of exponential type $M$ such that:

(i) The imbedding $W_{0}^{\text {i.p }}\left\llcorner E_{M}(\Omega)\right.$ is compact;

(ii) $M$ is superhomogeneous of degree $\sigma,+1>p$;

(iii) There are some $c_{1}>0$ and $K_{1}>0$ such that:

$$
\forall x \in \Omega, \quad \forall \zeta \in \mathbb{R}, \zeta g(x, \zeta) \leqslant c, M\left(\frac{\zeta}{K_{1}}\right) ;
$$

(iv) $\forall K>0, \lim _{\zeta \rightarrow \infty} \frac{g\left(x_{,} \zeta\right)}{M^{\prime}\left(\frac{\zeta}{K}\right)}=0$ uniformly in $x$ 
Then there is at least one positive solution $u \in \mathrm{W}_{0}^{1, p}(\Omega) \cap C^{1, a}(\Omega)$ of $(E)$.

\section{Example:}

Let $p=N=2 ; g(x, \zeta)=\zeta \sigma \exp (\zeta)$ with $\sigma>1,0<q<2$, and

$$
M(\zeta)=\mid \zeta j+1-r\left(e^{\zeta \zeta+r}-1\right) \text { with } q<r<2 .
$$

$r<2$ gives (i) [1]; $\zeta \rightarrow e^{|\zeta|^{r}}-1$ is superhomogeneous of degree $r$, whence (ii); (iii) is easy and $q<r$ gives (iv).

So, the equation:

$$
\Delta u+u^{\alpha} e^{u^{q}}=0
$$

has at least one positive solution $u \in \mathrm{W}_{0}^{1,2}(\Omega)$.

In a similar case TRUDINGER [15] proves that for any $m>0$, there exist $\lambda>0$ and $u>0$ such that $\int_{\Omega} G(., u)=m$ and

$$
\Delta u+\lambda g(x, u)=0
$$

Our method allows us to eliminate this $\lambda$.

We obtain the same results for the equation:

$$
\Delta_{\mathrm{p}} u+u^{\mathrm{a}} e^{u^{4}}=0
$$

where $p=N \geqslant 2, \sigma>p-1,0<q<\frac{p}{p-1}$

$J$ being defined in lemma 1 , the proof of Theorem 2 needs the following lemma.

\section{Lemma 3:}

If the hypothesis of Theorem 2 are satisfied, $J \in \mathrm{C}^{\prime}\left(\mathrm{W}_{0}^{1, p(\Omega)}(\Omega)\right.$ and satisfies the Palais Smale condition.

\section{Proof:}

Let $\left(u_{j}\right)$ be a bounded sequence in $\mathrm{W}_{0}^{\mathrm{l}, \mathrm{p}}(\Omega)$.

By (i) there is some $K>0$ such that: 


$$
\forall j, \int_{\Omega} M\left(\frac{u_{j}}{K}\right) \leqslant 1
$$

Let $c>0$ be such that $M^{*}\left(\frac{1}{\tau}\right)$ meas $(\Omega)<1$ and:

$$
\forall x \in \Omega, \forall \zeta \in \mathbb{R},|g(x, \zeta)| \leqslant \frac{c}{2}+\frac{1}{2} M\left(\frac{\zeta}{K}\right)
$$

We obtain:

(1) $\int_{\Omega} M^{*}\left[\frac{g\left(., u_{j}\right)}{c^{2}}\right] \leqslant \int_{\Omega} \frac{1}{2} M^{*}\left(\frac{1}{c}\right)+\int_{\Omega} \frac{1}{2} M\left(\frac{u_{j}}{K}\right) \leqslant 1$.

Let $u_{j}$ converges to $u$ in $W_{0}^{1, p}(\Omega)$. For sufficiently small $\delta$ and for meas $(A)<\delta$, we have:

$$
\begin{aligned}
& \int_{A} M^{*}\left[\frac{g\left(., u_{j}\right)}{c^{2}}\right] \\
& \leqslant \frac{1}{2} \mathrm{M}^{*}\left(\frac{1}{c}\right) \operatorname{meas}(A)+\frac{1}{4} \int_{A} M\left(\frac{u_{j}-u}{K}\right)+\frac{1}{4} \int_{A} M\left(\frac{u}{K}\right) \leqslant \varepsilon .
\end{aligned}
$$

$M^{*}\left[\frac{g(., u)-g(., u)}{c^{2}}\right]$ is then an equi-summable sequence and $\lim _{\mathrm{j} \rightarrow+\infty} \int_{\Omega} M^{*}\left[\frac{g\left(., u_{j}\right)-g(., \underline{u})}{c^{2}}\right]=0$

By (ii) $M^{*}$ satisfies the " $\Delta_{2}-$ condition" [11], so $\lim \left\|g\left(., u_{1}\right)-g(., u)\right\|_{M^{*}}=$ 0 ; whence $J \in \mathrm{C}^{1}\left(\mathbf{W}_{0}^{1, \mathrm{p}}(\Omega)\right)$.

Suppose now that $\left|J\left(u_{j}\right)\right| \leqslant K_{1}$ and $J^{\prime}\left(u_{j}\right) \rightarrow 0$. By lemma $1,\left\|u_{j}\right\|_{w^{1, p} \text { is boun- }}$ ded and, by (i), $u_{j}$ converges in $E_{M}(\Omega)$; by relation (1), $g\left(., u_{j}\right)$ converges for $\sigma\left(L_{M^{*}}, E_{M}\right)$. So the same proof than for lemma 2 shows that the Palais-Smale condition is satisfied.

\section{Proof of Theorem 2:}

Let us show that for $\|u\|_{w_{0}^{1, p}}=r$ sufficiently small we have $J(u) \geqslant \alpha>0$. 
By (iii) and (ii), we have

$$
\forall x \in \Omega, \forall \zeta \in \mathbb{R}, \forall h \in[0,1], G(x, \zeta) \leqslant c_{l} M\left(\frac{\zeta}{\mathrm{K}_{1}}\right) \leqslant h^{\sigma_{1}+1} M\left(\frac{\mathrm{K} \zeta}{\mathrm{K}_{1} h}\right)
$$

By (i)

$$
\forall u \in \mathrm{W}_{\mathrm{o}}^{\mathrm{l} \cdot \mathrm{p}}(\Omega),\|u\|_{M} \leqslant c\|u\|_{\mathrm{w}_{0}^{1, \mathrm{p}} \mathrm{p}}
$$

Whence for $\|u\|_{w_{0}^{1, p}}=r \leqslant \frac{K_{L}}{c K}$ and $h=\frac{c K r}{K_{l}}$ :

$\int_{\Omega} G(, u) \leqslant c_{l} \int_{\Omega} M\left(\frac{u}{K_{1}}\right) \leqslant c_{f} h^{\sigma_{1}+1} \int_{\Omega} \mathrm{M}\left(\frac{u}{c r}\right) \leqslant c_{t} h^{o_{1}+1}=c^{\prime}\|u\|_{\substack{w_{0}+1 \\ w_{0}^{1, p}}}$

The same proof than for Theorem 1 gives $t \in W_{0}^{1 . p}(\Omega), u \neq 0$, solution of (E). The end of the proof is a consequence of the following lemma. $\square$.

\section{Lemma 4:}

If all the hypothesis of Theorem 2 are satisfied, $u \in C^{\prime a}(\Omega)$.

\section{Proof:}

This proof is very similar to OTANI's one [9] (see also [13]). By (iii) there is some $s>1$ such that $u g(x, u) \in L^{s}(\Omega)$.

Consider the following sequences:

$$
\begin{aligned}
& q_{1}=2 p s^{*}=2 p s /(s-1) \\
& q_{k+1}=2\left(p+q_{k}\right) \\
& \theta=s^{*} q_{k}
\end{aligned}
$$

Multiplying $(E)$ by $|u|^{q_{k}} u$, we obtain:

$$
\begin{array}{r}
\left.\left(\frac{p}{p+q_{k}}\right)^{*} \int_{\Omega}|\nabla| u^{1+\frac{q_{k}}{p}}\right)\left.\right|^{p}=\int_{\Omega} u g(, u)|u|^{q_{k}} \\
\leqslant\|u g(, u)\|_{.}\left\|u^{q_{k}}\right\|_{*} \leqslant c\|u\|_{*}^{q_{k}}
\end{array}
$$

$M$ being of exponential type, $\mathrm{W}^{1 . \mathrm{p}}(\Omega) L^{2 N^{*}}(\Omega)$ and there is some $K$ such that: 


$$
\|u\|_{2 s^{*}\left(p+q_{k}\right)}^{p+q_{k}} \leqslant K^{p} \int_{\Omega}\left|\nabla\left(u^{1+\frac{q_{k}}{p}}\right)\right|^{p}
$$

We then obtain:

$$
\|u\|_{\theta_{k+1}}^{\theta_{k+1} / 2 s^{*}} \leqslant c\left(\frac{K\left(p+q_{k}\right)}{p}\right)^{p}\|u\|_{\theta_{k}}^{\theta_{k} / s^{*}}
$$

This formal proof can be made rigorous by using some regularized equation [13].

Observing that $p+q_{k} \leqslant 4^{k-1} 4 p s^{*}$, we get:

$$
\|u\|_{\theta_{k+1}}^{\theta_{k+1}} \leqslant c^{2 s^{*}}\left(4 K s^{*}\right)^{2 * s^{*}} 4^{2(k-1 p * * \|} u \|_{\theta_{k}}^{2 \theta_{k}}
$$

Let:

$E_{k}=\theta_{k} \log \|u\|_{\theta_{k}}$

$a=4^{2 p s^{\circ}}$

$b=\log \left[c^{2 *}\left(2 K s^{*}\right)^{20 *}\right]$

$r_{k}=b+(k-1) \log a$.

We then obtain:

$$
E_{k+1} \leqslant r_{k}+2 E_{k}
$$

Whence, following OTANI [9], we deduce:

$$
\|u\|_{\infty} \leqslant \overline{\lim }_{k \rightarrow+\infty} \exp \left(\frac{E_{k}}{\theta_{k}}\right)<+\infty
$$

So $u \in L^{\infty}(\Omega)$ and by TOLKSDORF's results $u \in C^{\prime a}(\Omega)$.

\section{A PARTICULAR CASE : $\Omega$ IS A BALL}

In the particular case when $\Omega$ is a ball and $N=p$, we can obtain radially symmetric solutions of $(E)$, for any growth of $g$ near infinity.

For simplicity we suppose that $g$ does not depend on $x$; we assume the following conditions: 
(H4) $g \in \mathrm{C}^{\mathrm{l}}(\mathbb{R}), g \geqslant 0$ and $g(0)=0$;

(H5) $\quad g$ and $g$ ' are non decreasing on $\mathbb{R}_{+}$;

(H6) $\lim _{t \rightarrow 0} \frac{g(t)}{t^{p-1}}=0$

\section{Theorem 3:}

Let $N=p \geqslant 2$ and let $g$ satisfy the conditions (H4), (H5), (H6). Then, there exists $R_{0}$ such that, for $R \geqslant R_{0}$, the equation

$$
\Delta_{p} u+g(u)=0 \text { in } \Omega=B(O, R)
$$

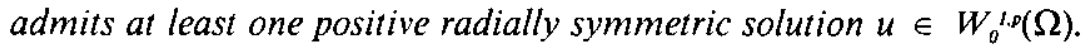

\section{Example:}

For any $\sigma>\max (1, p-1)$ and any $q>1, \mathrm{~g}(\zeta)=|\zeta|^{\sigma} \exp |\zeta|^{\circ}$ satisfies (H4), (H5), (H6).

Theorem 3 is a consequence of the following proposition. Let us consider the following system:

$(S)\left\{\begin{array}{l}v^{\prime}(x)=|w(x)|^{p^{*}-2} w(x) \\ w^{\prime}(x)=-\frac{e^{-x}}{p^{p}} g[v(x)]\end{array}\right.$

where $p^{*}=\frac{p}{p-1}$

Submitted to the conditions:

(L.C.) $\left\{\begin{array}{l}\lim _{x \rightarrow+\infty} v(x)=m \\ \lim _{x \rightarrow+\infty} w(x)=0 .\end{array}\right.$

\section{Proposition 2:}

Let $p \geqslant 2$ and let $g$ satisfy the conditions (H4), (H5), (H6). Then, for any $m>0,(S)+($ L.C.) admits one and only one solution $(v, w) ;$ there exists some $\boldsymbol{\alpha}=\theta(m) \in \mathbb{R}$ such that:

$$
v(\alpha)=0 \text { and } v>0 \text { on }] \alpha,+\infty]
$$


Moreover $\theta$ is continuous on $\mathbb{R}_{+}$and $\lim \theta(m)=-\infty$.

$$
m \rightarrow 0
$$

Proof:

Let us consider the following iterations;

$v_{0}=0$ and for $n \in \mathbb{N}$ :

$$
\begin{gathered}
w_{n}(x)=\int_{x}^{+\infty} \frac{e^{-t}}{p^{p}} g\left[v_{n}(t)\right] d t \\
v_{n+1}(x)=m-\int_{x}^{+\infty}\left|w_{n}(t)\right| p^{*-2} w_{n}(t) d t .
\end{gathered}
$$

We have:

$$
\begin{gathered}
w_{l}(x)=\frac{g(m)}{p^{p}} e^{-x}>w_{0}=0 \\
v_{2}(x)=m-\frac{g(m)}{p^{p}\left(p^{*}-1\right)} \exp \left[-\left(p^{*}-1\right) x\right]<m=v_{1}(x)
\end{gathered}
$$

There is some $M(m, p)$ such that:

$$
\begin{gathered}
\forall x \geqslant M(m, p), v_{2}(x) \geqslant \frac{m}{2}>v_{0}(x) \text { and } \\
w_{2}(x)=\int_{x}^{+\infty} \frac{e^{-t}}{p^{p}} g\left[v_{2}(t)\right] d t \geqslant \frac{f\left(\frac{m}{2}\right)}{p^{p}} e^{-x}
\end{gathered}
$$

By induction we can prove that for any $q \in \mathbb{N}, v_{2 q}$ is a nondecreasing sequence, $v_{2 q+1}$ is a noninncreasing sequence and $v_{2 q} \leqslant v_{2 q+1}$; whence for any $n$ we have either $v_{n} \leqslant v_{n+1}$, or $v_{n+1} \leqslant v_{n}$

Suppose that $n \geqslant 2$ and $v_{n} \leqslant v_{n+1}$; we have $w_{n} \leqslant w_{n+1}$ and $v_{n+2} \leqslant v_{n+1}$. $p^{*} \leqslant 2$ and $w_{n} \geqslant w_{2}$, whence :

$\left|w_{n+1}(t) \mathrm{p}^{\mathrm{p}^{*}-2} w_{n+1}(t)-\right| w_{n}(t)^{\mid \mathrm{p}^{*}-2} w_{n}(t) \leqslant\left(p^{*}-1\right) \mid w_{2}(t)^{\mathrm{p}^{*}-2}\left[w_{n+1}(t)-w_{n}(t)\right]$. 
We then obtain:

$0 \leqslant v_{n+1}(x)-v_{n+2}(x) \leqslant \frac{p^{*}-1}{p^{*}-2}\left(\frac{g\left(\frac{m}{2}\right)}{p^{p}}\right)^{p^{*-2}} \exp \left[-\left(p^{*}-2\right) x\right] \operatorname{Sup}_{t \in[x,+\infty]} \mid w_{n+1}(t)$ $-w_{n}(t)$

On the other hand, by (H5), we get:

$$
0 \leqslant w_{n+1}(x)-w_{n}(x) \leqslant \frac{g^{\prime}(m) e^{-x}}{p^{p}} \operatorname{Sup}_{t \in[x,+\infty]}\left|v_{n+1}(x)-v_{n}(x)\right| .
$$

Therefore:

$$
\operatorname{Sup}_{t \in[x,+\infty]}\left|v_{n+2}(x)-v_{n+1}(x)\right| \leqslant c(x) \operatorname{Sup}_{t \in[x,+\infty]}\left|v_{n+1}(t)-v_{n}(t)\right| .
$$

And, for $x \geqslant M_{f}(m, p) \geqslant M(m, p)$, we have:

$$
\alpha(x)=\frac{p^{*}-1}{p^{*}-2}\left(\frac{g\left(\frac{m}{2}\right)}{p^{\natural}}\right)^{p^{*-2}} \frac{g^{\prime}(m)}{p^{\natural}} \exp \left[-\left(p^{*}-1\right) x\right]<1
$$

By Picard's theorem we obtain a unique solution $(v, w)$ of $(S)+($ L.C) for $x \geqslant M_{1}(m, p)$. By classical differential equations theory this solution can be continued for $x<M_{i}(m, p)$. Since $v$ has increasing gradient, it has a last zero at a point $x=\alpha=\theta(m)$.

Let us set:

$$
H(x, m)=\int_{x}^{+\infty}|w(t, m)|^{p^{*}-2} w(t, m) d t-m
$$

$\frac{\partial H}{\partial x}(\alpha, m) \neq 0$ and by implicit functions theorem $\theta$ is continuous.

For $x \in] \alpha,+\infty$ ], we have $0<v(x) \leqslant m$, whence:

$$
w(x) \leqslant \int_{x}^{+\infty} \frac{e^{-1}}{p^{p}} g(m)=\frac{e^{-x}}{p^{p}} g(m)
$$




$$
\begin{aligned}
& m=\int_{\alpha}^{+\infty} v^{\prime}(x) d x \leqslant\left(\frac{g(m)}{p^{p}}\right)^{p^{*}-1} \frac{e^{-\left(p^{*}-1\right) a}}{p^{*}-1} \\
& \lim _{m \rightarrow 0}\left(p^{*}-1\right) p^{p} e^{\left(p^{*}-1\right)(m)} \leqslant \lim _{m \rightarrow 0}\left(\frac{g(m)}{m^{p-1}}\right)^{p^{*-1}}=0
\end{aligned}
$$

whence $\lim \theta(m)=-\infty$. $\square$

$$
m \rightarrow 0
$$

\section{Proof of Theorem 3:}

By proposition 2, there is some $\alpha_{0}$ such that for any $a=-p \log R \leqslant \alpha_{0}$, (S) + (L.C.) has one and only one solution such that $v(\alpha, m)=0$.

The change of variable $x=-p \log r, v(x)=\varphi(r)$ transforms (S) into the equation:

$$
\frac{d}{d r}\left\{r r \varphi^{\prime}(r) \mid p-2 r \varphi^{\prime}(r)\right\}+r^{\rho-1} g[\varphi(r)]=0
$$

which is the radial form of the equation $(E)$, with boundary condition $\varphi(R)=0$.

Remark: The deep study of the case $p=2$ made by HEMPEL [5] and NEHARI [6] shows that there is no hope to find a solution of (E) for any $R$, if the growth of $g$ has no bound when $u \rightarrow+\infty$.

\section{References}

[1] R.A. ADAmS, Sobolev Spaces; Academic Press, 1975.

[2] C. BANDle and L. A. Peletier, Problèmes de Dirichlet non-liméaires dans des anneaux; C.R. Acad. Sc. Paris t. 303, Serie I n. ${ }^{\circ}$, 1986, 181-184.

[3] X. GARAIZAR, Existence of positive radial solutions for semilinear elliptic equations in the annulus; Journal of Diff, eq. 70, 1987, 69-92.

[4] J.A. HEMPEL, Superlinear variational boundary value problems and nonuniqueness; Thesis, Univ. New England, 1970.

[5] J.A. HEMPEL, On a superlinear differential equation; Indiana Univ. Math. Journal, vol. $26 \mathrm{n} .^{\circ} 2,1977,265-275$.

[6] Z. NEHARI, On a class of nonlinear second order differential equations; Trans. Amer. Math. Soc. 95, 1960, 101-123.

[7] W.M. NI and J. SERRIN, Existence and non-existence theorems for ground 
states for quasilinear partial differential equations; Proc. Acad. Lincei (to appear).

[8] L. NIRENBerg, Variational and topological methods, in nonlinear problems; Bulletin of the A.M.S., vol. 4 n. ${ }^{\circ} 3,1981,267-302$.

[9] M. OTANI, Existence and non-existence of non trivial solutions of some nonlinear degenerate elliptic equations; J. Funct. Analysis, vol. 76 n. ${ }^{\circ} 1$, 1988, 140-159.

[10] S.I, POHOzAEv, Eigenfunction of the equation $\Delta u+\lambda f(u)=0$; Soviet. Math. Dokl n. ${ }^{\circ} 6,1965,1408-1411$.

[11] F. DE THELIN, Régularité de la solution d'une équation fortement (ou faiblement) non linéaire; Annales Fac. Sciences Toulouse, vol. 2, 19.80, 249-281.

[12] F. DE THELIN, Quelques résultats d'exisence et de non existence pour une E.D.P. elliptique non linéaire; C.R. Acad. Sc. Paris, t. 299, Serie I n. ${ }^{\circ} 18$, 1984, $911-914$.

[13] F. DE THELIN, Résultats d'existence et de non existence pour la solution positive et bornée d'une E.D.P. elliptique non linéaire; Annales Fac. Sciences Toulouse, vol. VIII n. ${ }^{\circ} 3,1987,373-389$.

[14] P. TOLKSDORF, On the Direchlet problem for quasilinear equations in domains with conical boundary points; Comm. P.D.E., n. ${ }^{\circ} 8,1983,773-817$.

[15] N.S. TRUDINGER, On imbeddings into Orlicz spaces and some applications; J. of Math. and Mech. vol. 17 n. ${ }^{\circ}$ 5, 1967, 473-483.

[16] J.L. VAZQUEZ, A strong maximum principle for quasilinear elliptic equations; Appl. Math. and Optimization, n. ${ }^{\circ} 12,1984,191-202$.

Université Paul Sabatier

Recibido: 17 noviembre 1988

31062 Toulouse

FRANCE 